\title{
Ligamentum teres tendinopathy and tears
}

\author{
Matthew J. Kraeutler ${ }^{1}$ \\ Tigran Garabekyan² \\ Cecilia Pascual-Garrido ${ }^{1}$ \\ Omer Mei-Dan'1 \\ 1 University of Colorado School of Medicine \\ 2 Southern California Hip Institute \\ Corresponding author: \\ Omer Mei-Dan \\ Hip Preservation/Sports Medicine/Orthopedics \\ University of Colorado \\ 12631 East 17th Avenue \\ Mail Stop B202, Room L15-4505 \\ Aurora, Colorado 80045 (USA) \\ E-mail: omer.meidan@ucdenver.edu
}

\section{Summary}

Background: The ligamentum teres (LT) consists of two bands that originate on the ischial and pubic sides of the acetabular notch and insert on the fovea capitis of the femoral head. Recent studies have established the LT as an important hip stabilizer in a squatting position, particularly in patients with osseous instability.

Purpose: This review aims to concisely present the literature on LT tendinopathy and tears in order to guide health care professionals in the appropriate diagnosis and treatment of these disorders.

Methods: We reviewed the literature on the diagnosis and surgical management of ligamentum teres tendinopathy and tears.

Conclusions: The ligamentum teres is an important stabilizer to the hip joint, particularly with hip flexion and external rotation. Older age and acetabular bony pathomorphology are two of the known risk factors for LT tears. Symptoms of LT tendinopathy are largely non-specific, mimicking a wide range of other hip disorders including impingement and instability. Debridement of LT tears or reactive tissue has been reported with good outcomes, with more recent studies describing reconstruction of a completely torn, nonfunctional, or absent LT using various graft sources including synthetic grafts, autografts, and allografts.

Level of evidence: II.

KEY WORDS: hip arthroscopy, ligamentum teres, tendinopathy.

\section{Introduction}

The ligamentum teres (LT) consists of two bands that originate on the ischial and pubic sides of the acetabular notch and blend with the transverse acetabular ligament between these two attachment sites. The two bands insert on the fovea capitis of the femoral head $^{1}$. The LT starts as a flat ligament at its acetabular origin and transitions to a round or tubular ligament at its attachment on the fovea capitis ${ }^{2}$. The LT is composed of collagen types I, III, and IV 2 and is surrounded by a layer of synovium and contains small arteries (including the artery femoris capitis), veins, and nerve bundles ${ }^{3}$. The mean length of the LT is between 30 and $35 \mathrm{~mm}^{1,4}$. At its yield and failure points, the mean length of the LT is approximately $38 \mathrm{~mm}$ and $53 \mathrm{~mm}$, respectively ${ }^{4}$

Traditionally, there has been no consensus on the role of the ligamentum teres in providing stability to the hip joint ${ }^{5}$. However, recently it has been shown that the LT serves as an important stabilizer with the hips in a squatting position (i.e. hip flexion and external rotation) as well as when attempting to cross the involved leg behind the other (i.e. hip extension and internal rotation $)^{6,7}$. These two positions create maximum tension on the ligamentum teres ${ }^{6}$. The stability imposed by the LT is particularly important in those with osseous instability, such as inferior acetabular insufficiency, borderline or frank hip dysplasia, or some forms of femoroacetabular impingement $(\mathrm{FAI})^{6}$. Although animal models have shown an increased risk of hip dislocation with complete rupture of the LT, this phenomenon has not been proven in humans ${ }^{2}$. Absence of the ligamentum teres has been shown to occur in approximately $19 \%$ of pediatric patients requiring open reduction for developmental dysplasia of the hip $(\mathrm{DDH})^{8}$. Another common finding in patients with $\mathrm{DDH}$ is a hypertrophic $\mathrm{LT}^{9,10}$

This article submits to the ethical standards of the journal11.

\section{Ligamentum teres tendinopathy}

In 1997, Gray and Villar ${ }^{12}$ identified 20 patients with ligamentum teres pathology found during hip arthroscopy. Based on their findings, the Authors instituted a classification system that includes three types of pathology: complete rupture of the LT (Group 1 ), partial rupture of the LT (Group 2), and degenerate ligamentum teres (Group 3). Group 3 is charac- 
terized by frayed and degenerative changes in the ligament, with most patients demonstrating symptoms of hip osteoarthritis.

The prevalence of LT tears has been analyzed in a number of studies. In a case series of healthy volunteers with no history of hip symptoms or surgery, Register et al. ${ }^{13}$ found a $2.2 \%$ prevalence of LT tears (1 of 45 patients) based on 3.0-tesla magnetic resonance imaging (MRI) scans. Botser et al. ${ }^{14}$ found LT tears in 284 of 558 patients $(51 \%)$ undergoing hip arthroscopy for various pathologies. Overall, $3.7 \%$ of patients had a complete rupture, $43 \%$ had a partial tear, and $4.5 \%$ had a degenerative tear. In another study ${ }^{15}$, the ligamentum teres was found to be normal in 152 of 184 (83\%) patients undergoing hip arthroscopy for various hip pathologies. However, complete ruptures were found to be present in 7 patients $(3.8 \%)$, a prevalence nearly identical to that cited by Botser et al. ${ }^{14}$.

The various risk factors for LT tears have also been investigated (Table I). Cerezal ${ }^{15}$ found that 25 out of 32 patients with partial or complete LT tears exhibited femoroacetabular impingement (FAI), in addition to 3 with adult hip dysplasia, 3 with hip instability, and 1 with traumatic tearing. Botser et al. ${ }^{14}$ found that patients with LT tears were significantly older at the time of arthroscopy, had worse preoperative functional scores, and had greater range of motion. Patients with LT tears did not have a significantly different visual analog scale (VAS) pain score compared with those without LT tears. In a series of 463 hips, Domb et al. ${ }^{16}$ found that patients with and without LT tears had an average age of 38 and 33 years, respectively. In addition, acetabular bony morphology was found to represent a risk factor for LT tears ${ }^{16}$. Specifically, hips with low lateral coverage index ( $\mathrm{LCl}$ ) values were 1.74 times more likely to have a tear of the ligamentum teres compared with high LCI hips. The lateral coverage index is calculated as the difference between the lateral center edge (LCE) angle and the acetabular inclination (AI) angle, and thus lower $\mathrm{LCI}$ values refer to lower LCE angles, which are indicative of borderline or frank hip dysplasia. In other words, Domb found that patients with radiographic features of hip dysplasia were more likely to have an LT tear. Furthermore, patients with tears had less acetabular retroversion in Domb's study ${ }^{16}$. Finally, there may also be an as- sociation between the articular cartilage damage pattern seen in patients with FAI and risk of LT tears. In a case series of 77 consecutive patients undergoing hip arthroscopy for FAI, Kaya et al. ${ }^{17}$ found that patients with LT tears were more likely to have articular cartilage damage in the inferior middle portion of the acetabulum and the apex of the femoral head when compared with patients with an intact LT.

\section{Diagnosis}

The mechanisms of complete ligamentum teres tears include traumatic hip dislocation and iatrogenic injury occurring during open surgical dislocation of the hip ${ }^{2}$. Repetitive microtrauma induced by hyperlaxity or instability (e.g., borderline and frank hip dysplasia) has also been proposed as a cause of complete LT tears. Partial tears are much more common and may be caused by chronic irritation or subtle subluxation from bony or functional (such as Ehlers-Danlos Syndrome) borderline hip dysplasia or a large Cam lesion in patients with FAI. In a biomechanical model involving 12 cadavers, Philippon et al. ${ }^{4}$ found that the most common mechanism of failure was intrasubstance tearing at the fovea capitis followed by acetabular peel-off. Avulsion at the fovea capitis occurred in only one case.

Patients with symptoms indicative of ligamentum teres pathology should undergo a history and physical examination. The most common symptoms are localized groin pain, hip instability, altered range of motion, and pain with log rolling of the hip and passive internal rotation at $90^{\circ}$ of flexion (Table II) ${ }^{18}$. Persistent hip or groin pain may be related to the loss of stability induced by a ruptured or degenerative ligamentum teres ${ }^{7}$. Global instability of the hip may be assessed via the axial traction apprehension test ${ }^{19}$. With the patient supine, an assistant stabilizes the patient's pelvis while manual axial traction is applied to the leg in an attempt to distract the joint. The test is considered positive if the patient experiences apprehension during the maneuver.

Recently described by O'Donnell et al. ${ }^{20}$, the "LT test" is performed with the patient in 70 degrees of hip flexion and $30^{\circ}$ short of full hip abduction. The examiner then internally and externally rotates the hip to its

Table I. Risk factors associated with tears of the ligamentum teres.

\begin{tabular}{|c|c|}
\hline Risk factor & Reference(s) \\
\hline Older age & Botser et al, $2011^{14}$, Domb et al, $2013^{16}$ \\
\hline Worse preoperative functional scores & Botser et al, $2011^{14}$ \\
\hline Greater hip range of motion & Botser et al, $2011^{14}$ \\
\hline Low lateral coverage index (LCI = LCE - Al) & Domb et al, $2013^{16}$ \\
\hline Less acetabular retroversion Domb et al, $2013^{16}$ & \\
\hline
\end{tabular}

LCE = lateral center edge angle, $\mathrm{Al}=$ acetabular inclination angle 
Table II. Symptoms of ligamentum teres tears.

\begin{tabular}{ll}
\hline Symptom & Possible underlying pathology \\
\hline Localized groin pain & Hip OA, FAI \\
Hip instability & Hip dysplasia \\
Altered range of motion & Hip OA, FAI, dysplasia \\
Pain with log roll test & Hip OA, FAI \\
Pain with passive internal rotation at 90 degrees of flexion & FAI \\
\hline
\end{tabular}

$\mathrm{OA}=$ osteoarthritis, $\mathrm{FAI}=$ femoroacetabular impingement

limits. A positive test is indicated by pain with either internal or external rotation. In a series of 75 patients with two examiners performing the LT test on each patient, the sensitivity and specificity for predicting an LT tear was $90 \%$ and $85 \%$, respectively, based on arthroscopic findings. Furthermore, the efficacy of the test was found to be unaffected by the presence of a partial versus a degenerative tear. However, both examiners found the test to be negative in the one patient with a complete rupture of the LT, and thus this test is not effective in detecting complete LT tears.

Imaging may be used to further assess for the presence of a ligamentum teres rupture. MRI has been shown to have lower sensitivity and specificity in detecting partial LT tears compared with magnetic resonance arthrography (MRA) ${ }^{21}$. Based on arthroscopic correlation, Schmaranzer et al. ${ }^{22}$ found that MRA demonstrated $90 \%$ and $72 \%$ sensitivity with and without the use of hip traction. Cerezal et al. ${ }^{15}$ found a similar sensitivity (92\%) with MRA and leg traction for complete LT tears, though the sensitivity of highgrade partial tears was only $66 \%$ in this study.

\section{Surgical management}

Both debridement and reconstruction have been described in the surgical management of LT tears. These two procedures are typically indicated for two separate groups of patients. As mentioned above, LT tears of varying degrees are a common finding during hip arthroscopy. Patients with FAl often experience chronic LT tendinopathy or a partial tear. In these patients, minimal radiofrequency debridement is necessary to denervate the inflamed tissue, while surgical correction of the FAl should prevent the accumulation of additional tears. On the other hand, LT reconstruction is typically reserved only for patients with hip instability when a bony realignment procedure (e.g. periacetabular osteotomy or derotational femoral osteotomy) is not indicated.

Both LT debridement and reconstruction have demonstrated improved subjective outcome scores and range of motion and a high rate of return to sport ${ }^{18}$. In one study $^{23}, 29$ patients underwent arthroscopic debridement for an isolated LT rupture. At 12 months follow- up, the mean Modified Harris Hip Score (MHHS) and Non-Arthritic Hip Score (NAHS) had improved significantly compared to preoperatively. However, five patients $(17 \%)$ developed recurrent symptoms after surgery that went unresolved with conservative treatment. These five patients required a second arthroscopy at a mean follow-up of 9 months. All five patients were found to have recurrent partial tears that required re-debridement and capsular tightening, which resulted in 10-15 degree loss of external rotation which had been maintained through follow-up.

Amenabar et al. ${ }^{24}$ performed a prospective case series that included 27 partial LT tears in 26 patients. Patients were treated with arthroscopic debridement with radiofrequency and anterior capsular tightening. At an average follow-up of 32 months, MHHS and NAHS scores improved significantly compared to preoperatively. No revision surgery was required for recurrent tears.

\section{Reconstruction of the ligamentum teres}

Ligamentum teres reconstruction is typically indicated only for complete ligament tears. Reconstruction has been described with the use of a synthetic graft (the Ligament Augmentation \& Reconstruction System [LARS], Arcsur-Tille, France) ${ }^{7}$, allografts $^{25}$, a doublestranded semitendinosus autograft ${ }^{26}$, and an iliotibial (IT) band tendon autograft ${ }^{19}$. In the only case series reporting outcomes of LT reconstruction, Philippon et al. ${ }^{19}$ performed arthroscopic reconstruction of the LT using an IT band autograft in four patients. All patients reported resolution of symptoms and had returned to sport at a mean follow-up of 31 months. However, three of the four patients required additional procedures postoperatively: a resurfacing arthroplasty, lysis of adhesions, and an iliopsoas tendon release, respectively.

The majority of techniques for LT reconstruction employ femoral tunnel drilling through the peritrochanteric region and differ mainly with regard to graft type or acetabular fixation. In contrast, our group recently described an arthroscopic technique for reconstruction of the ligamentum teres with graft fixation at the femoral head-neck junction ${ }^{25}$. After confirming complete tear of the LT, the stump is debrided with a combination of a radiofrequency ablator 
(Super MultiVac 50, Smith \& Nephew, Andover, MA) and a shaver (4.5-mm 30 angled double bite, Stryker, Kalamazoo, MI). Note that access to the cotyloid fossa may be facilitated by waiting until the femoral tunnel has been drilled, allowing for transtunnel instrumentation.

While the surgeon carries out the diagnostic portion of the procedure, an assistant prepares the LT graft on the back table using either a single-stranded tibialis posterior or double-stranded semitendinosus allograft with a diameter of approximately 6 to $8 \mathrm{~mm}$. Recent reports of allograft resorption ${ }^{27}$ have led to an increased use of autograft, in which case the iliotibial band or quadriceps is preferred. The graft is tabularized using vicryl sutures and pretensioned on the Graftmaster (Acufex Graftmaster III, Smith \& Nephew) for approximately 20 minutes.

Before drilling the femoral tunnel, traction is released and drilling trajectory is assessed by using an 18gauge spinal needle under biplanar fluoroscopy. The goal is to start at the lower head-neck junction (distal to the cam resection line) and exit centrally in the fovea (Figure 1). After advancing a 2.3-mm guidewire (Arthrex, Naples, FL) toward the fovea under fluoroscopy, traction is gently reapplied before breaching the far cortex, to avoid plunging into the cotyloid fossa. A cannulated $5.0-\mathrm{mm}$ reamer (Arthrex) is advanced to the far cortex, without penetrating into the hip joint. Traction is again gently reapplied and drilling of the far cortex is completed.

After debriding the native LT stump, the posteroinferior bony bed of the cotyloid fossa is decorticated with a burr (4-mm hip burr, Stryker) to promote graft healing. It is important to have optimal trajectory while drilling for anchor placement in the medial acetabular wall28. This is achieved by choosing between the

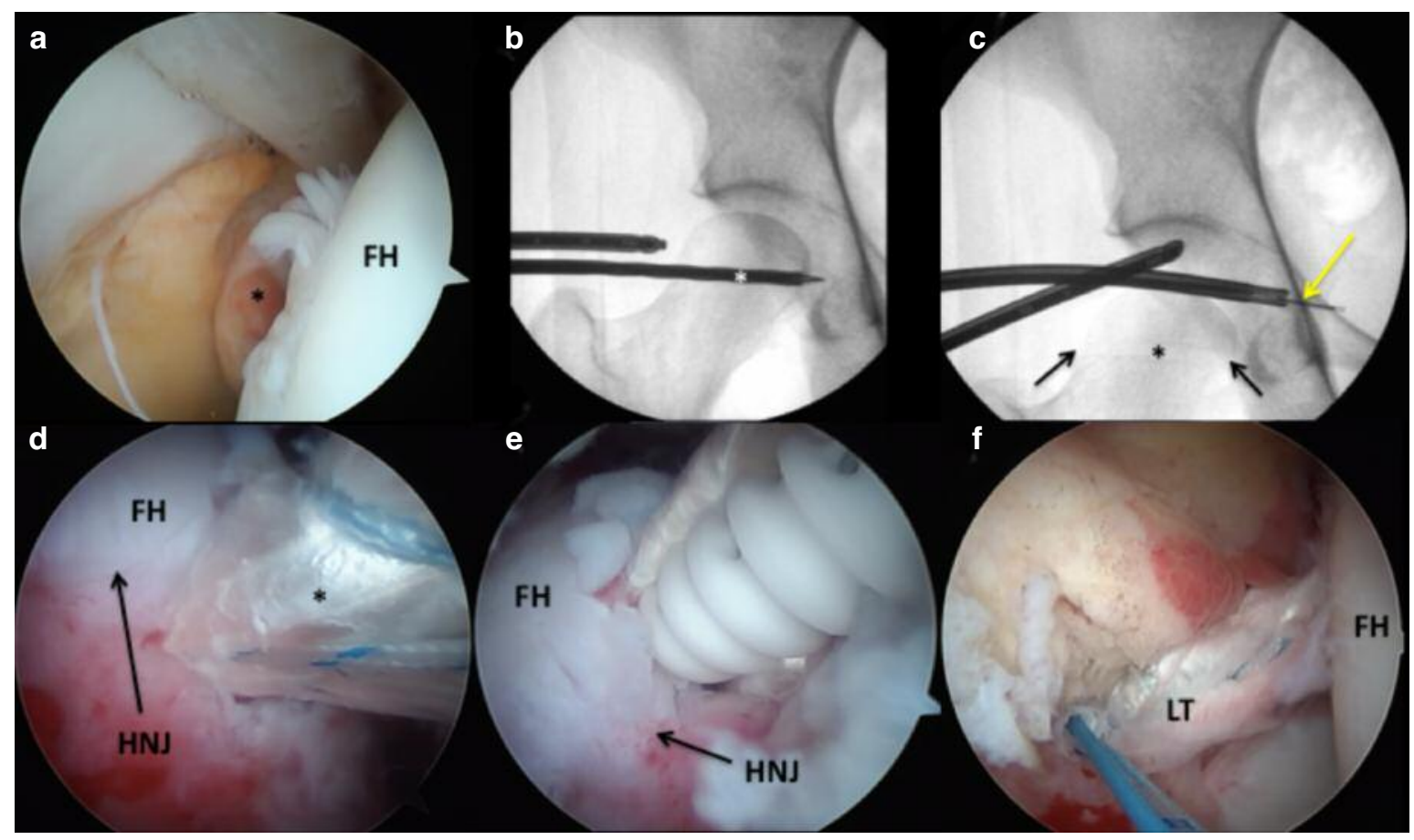

Figure 1 a-f. (a) Arthroscopic view of the central compartment of the right hip with the 70 degree arthroscope inserted through the mid-trochanteric portal, aimed at the pulvinar. The ligamentum teres (LT) stump (black asterisk) is seen, indicating a complete tear. (b) Anteroposterior fluoroscopic image of the right hip showing drilling of the femoral tunnel. The white asterisk denotes a 5.0-mm cannulated reamer, exiting at the level of the fovea. (c) Anteroposterior fluoroscopic view of the right hip showing proper placement of an all-suture anchor $(2.3 \mathrm{~mm}$ Iconix, No. 5 ultrastrength wire, Stryker) in the cotyloid fossa. The inserter must breach the pelvic brim by approximately 6 to $8 \mathrm{~mm}$ to allow for intrapelvic placement of the all-suture anchor, with aperture fixation against the strong medial wall cortical bone (yellow arrow). The previously drilled femoral head tunnel is visible (black asterisk) with the two black arrows delineating the point of entry at the femoral head-neck junction and exit at the fovea. (d) Arthroscopic view of the peripheral compartment of the right hip with the 70 degree arthroscope inserted through the mid-trochanteric portal, visualizing the anterior femoral head-neck junction. The LT graft (black asterisk) may be seen during passage into the femoral tunnel. (e) Arthroscopic view of the peripheral compartment of the right hip with the 70 degree arthroscope inserted through the mid-trochanteric portal, visualizing the anterior femoral headneck junction. A 4.75-mm biotenodesis screw (Arthrex) is seen advancing over a guidewire to secure the LT graft distally within the femoral tunnel. The hip is positioned in extension and external rotation during distal graft fixation. (f) Arthroscopic view of the central compartment of the right hip with the 70 degree arthroscope inserted through the mid-trochanteric portal, visualizing the contents of the pulvinar. The new LT graft is seen in anatomic position with good tension. FH = femoral head, $\mathrm{HNJ}=$ head-neck junction. 
femoral tunnel and either of the established arthroscopic portals and using curved drill guides (Iconix, $12^{\circ}$ and $25^{\circ}$ curved drill guides, Stryker). The first anchor is drilled at the anticipated center of the reconstructed LT, using a 2.3-mm double-loaded all-suture anchor (Iconix, No. 5 ultrastrength wire, Stryker) aiming the curved guide posteriorly to avoid the external iliac vessels. We prefer not to deploy the anchor within the tunnel as the tunnel length is often short resulting in anchor pullout or intra-articular protrusion. A second anchor is placed approximately $5 \mathrm{~mm}$ away from the first in a similar fashion. A divergent drill trajectory may be achieved either by rotating the curved drill guide or by changing the femoral rotation.

The graft is then inserted into the joint with the aid of the central acetabular anchor (Figure 2). Once the graft has been apposed to the acetabular insertion site, both suture limbs are retrieved from the same arthroscopic portal and a knot is tied to secure the graft. A Speedstitch device (ArthroCare, Austin, TX) is then used to pass the other three sutures (one from the first anchor and two from the additional anchor) through the base of the graft, allowing for increased surface contact between the graft and the bony bed. The Speedstitch is advantageous for this step as it acts both as a grasper and suture passer, allowing accurate suture placement with relative ease.

After securing the graft to the cotyloid fossa, its position is assessed at varying degrees of rotation under traction. Traction is then released and the leg placed in slight extension and extreme external rotation (50$60^{\circ}$ ) before securing the femoral side with a $4.75 \mathrm{~mm}$ $\times 15 \mathrm{~mm}$ bio-tenodesis screw (Arthrex). Excess graft is cut flush at the level of the femoral tunnel, taking care to avoid a bulky stump which may impinge with flexion and internal rotation. The hip is irrigated and closure carried out in a standard fashion.

\section{Postoperative rehabilitation}

Following arthroscopic $\mathrm{LT}$ reconstruction as described above ${ }^{25}$, a brace (X-Act ROM Hip Brace, DJO
Global, Vista, CA) is used to avoid hip hyperextension and external rotation. Physical therapy is begun immediately in the brace with passive range of motion to avoid intra-articular adhesions. "Toe-touch" weight bearing is employed for the first two weeks, with partial weight bearing with the brace for an additional four weeks. Return to play is allowed at 5 months postoperatively.

Lindner et al. ${ }^{26}$ describe a similar rehabilitation protocol following LT reconstruction with semitendinosus autograft or allograft. Foot-flat weight bearing is restricted to $20 \mathrm{lb}$ for the first six weeks postoperatively, with a hip brace locked at $0-90^{\circ} \mathrm{s}$ of hip flexion during this time.

\section{Conclusions}

Although there has been some debate as to the role that the ligamentum teres plays in providing hip stability, recently it has been shown that the LT is an important stabilizer to the hip joint, particularly with hip flexion and external rotation. The most common classification system of LT tendinopathy is that of Gray and Villar ${ }^{12}$, who categorized patients into three groups: those with a complete tear of the ligamentum teres, those with a partial tear, and those with a degenerate LT. Older age and acetabular bony morphology are two of the known risk factors for LT tears. Symptoms of LT tendinopathy may be broad, though hip instability or hip/groin pain are the most common symptoms in these patients.

In general, there are two cohorts of patients treated for tears of the ligamentum teres. The first group is the patient with FAl or hip osteoarthritis with chronic LT tendinopathy or a partial LT tear in which simple debridement is indicated. The second group includes patients with hip instability who are more likely to experience a complete tear of the LT. In these patients, $\mathrm{LT}$ reconstruction is reserved for cases when an osteotomy procedure to correct the hip dysplasia is not indicated. The literature on surgical management of LT tendinopathy is limited and mainly includes case

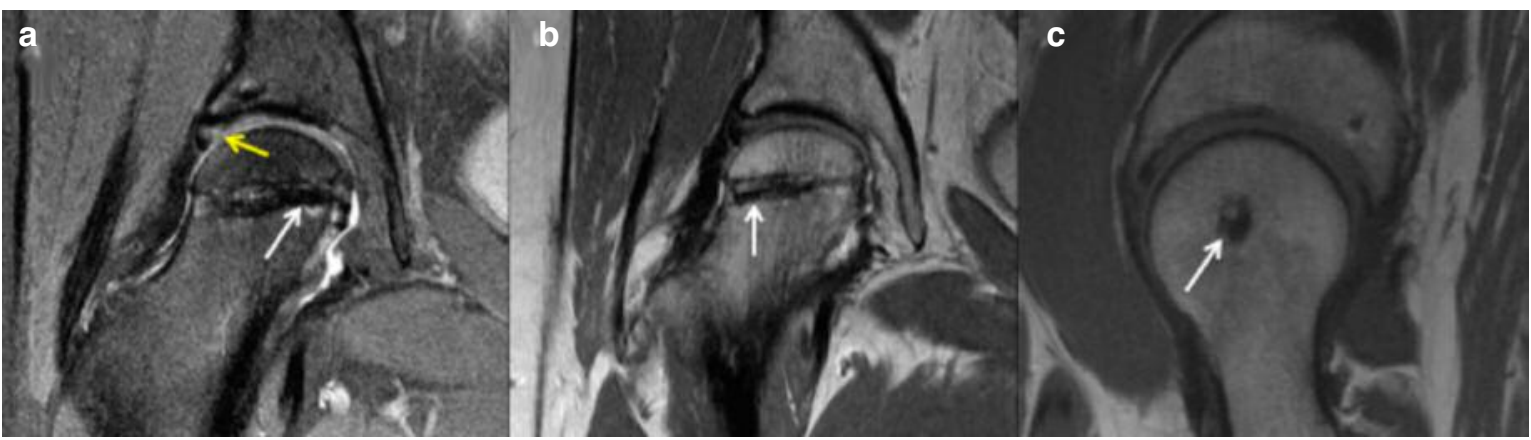

Figure 2 a-c. (a) Postoperative T2 coronal magnetic resonance imaging (MRI) of the right hip showing the ligamentum teres (LT) graft within the tunnel (white arrow). Signal intensity is seen in the chondrolabral junction (yellow arrow), indicating healing response after labral repair. (b) Postoperative T1 coronal MRI showing a well-seated biotenodesis screw $(4.75 \times 15$ $\mathrm{mm}$, Arthrex) within the femoral head (white arrow), securing the LT graft. (c) Postoperative T1 sagittal MRI showing centrally positioned graft tunnel (white arrow). 
reports and small case series. Although debridement of the ligamentum teres has been described with good outcomes, more recent reports have described reconstruction of the LT using various graft sources, including synthetic grafts, autografts, and allografts. Our group has described a novel technique of LT reconstruction using a single-stranded tibialis posterior or double-stranded semitendinosus allograft with graft fixation at the femoral head-neck junction.

Future studies should seek to determine a reliable prevalence of LT tears among patients complaining of hip instability or pain, as current studies have reported conflicting data. In addition, further research should seek to report outcomes in larger case series of patients to determine the appropriate graft type and fixation methods for reconstruction of the ligamentum teres.

\section{References}

1. Bardakos NV, Villar RN. The ligamentum teres of the adult hip. J Bone Joint Surg Br. 2009;91:8-15.

2. O'Donnell JM, Pritchard M, Salas AP, Singh PJ. The ligamentum teres-its increasing importance. J Hip Pres Surg. 2014;1:3-11.

3. Dehao BW, Bing TK, Young JL. Understanding the ligamentum teres of the hip: a histological study. Acta Ortop Bras. 2015;23:29-33.

4. Philippon MJ, Rasmussen MT, Turnbull TL, et al. Structural properties of the native ligamentum teres. Orthop J Sports Med. 2014;2:2325967114561962.

5. Martin HD, Savage A, Braly BA, Palmer IJ, Beall DP, Kelly B. The function of the hip capsular ligaments: a quantitative report. Arthroscopy. 2008;24:188-195.

6. Martin RL, Palmer I, Martin HD. Ligamentum teres: a functional description and potential clinical relevance. Knee Surg Sports Traumatol Arthrosc. 2012;20:1209-1214.

7. Simpson JM, Field RE, Villar RN. Arthroscopic reconstruction of the ligamentum teres. Arthroscopy. 2011;27:436-441.

8. Li T, Zhang M, Wang H, Wang Y. Absence of ligamentum teres in developmental dysplasia of the hip. J Pediatr Orthop. 2015;35:708-711.

9. Bulut O, Oztürk H, Tezeren G, Bulut S. Arthroscopic-assisted surgical treatment for developmental dislocation of the hip. Arthroscopy. 2005;21:574-579.

10. Eberhardt O, Fernandez FF, Wirth T. Arthroscopic reduction of the dislocated hip in infants. J Bone Joint Surg Br. 2012;94: 842-847.

11. Padulo J, Oliva F, Frizziero A, Maffulli N. Muscles, Ligaments and Tendons Journal. Basic principles and recommendations in clinical and field science research: 2016 Update. MLTJ. 2016;6(1):1-5.

12. Gray AJ, Villar RN. The ligamentum teres of the hip: an arthro- scopic classification of its pathology. Arthroscopy. 1997;13:575-578.

13. Register B, Pennock AT, Ho CP, Strickland CD, Lawand A, Philippon MJ. Prevalence of abnormal hip findings in asymptomatic participants: a prospective, blinded study. Am J Sports Med. 2012;40:2720-2724.

14. Botser IB, Martin DE, Stout CE, Domb BG. Tears of the ligamentum teres: prevalence in hip arthroscopy using 2 classification systems. Am J Sports Med. 2011;39 Suppl:117S-125S.

15. Cerezal L, Carro LP, Llorca J, et al. Usefulness of MR arthrography of the hip with leg traction in the evaluation of ligamentum teres injuries. Skeletal Radiol. 2015;44:1585-1595.

16. Domb BG, Martin DE, Botser IB. Risk factors for ligamentum teres tears. Arthroscopy. 2013;29:64-73.

17. Kaya M, Suziki T, Minowa T, Yamashita T. Ligamentum teres injury is associated with the articular damage pattern in patients with femoroacetabular impingement. Arthroscopy. 2014;30:1582-1587.

18. de SA D, Phillips M, Philippon MJ, Letkemann S, Simunovic N, Ayeni OR. Ligamentum teres injuries of the hip: a systematic review examining surgical indications, treatment options, and outcomes. Arthroscopy. 2014;30(12):1634-1641.

19. Philippon MJ, Pennock A, Gaskill TR. Arthroscopic reconstruction of the ligamentum teres: technique and early outcomes. J Bone Joint Surg Br. 2012;94:1494-1498.

20. O'Donnell J, Economopoulos K, Singh P, Bates D, Pritchard $M$. The ligamentum teres test: a novel and effective test in diagnosing tears of the ligamentum teres. Am J Sports Med. 2014;42:138-143.

21. Datir A, Xing M, Kang J, et al. Diagnostic utility of MRI and MR arthrography for detection of ligamentum teres tears: a retrospective analysis of 187 patients with hip pain. AJR Am J Roentgenol. 2014;203:418-423.

22. Schmaranzer $F$, Klauser $A$, Kogler $M$, et al. MR arthrography of the hip with and without leg traction: Assessing the diagnostic performance in detection of ligamentum teres lesions with arthroscopic correlation. Eur J Radiol. 2016;85:489-497.

23. Haviv B, O'Donnell J. Arthroscopic debridement of the isolated ligamentum teres rupture. Knee Surg Sports Traumatol Arthrosc. 2011;19:1510-1513.

24. Amenabar T, O'Donnell J. Successful treatment of isolated, partial thickness ligamentum teres (LT) tears with debridement and capsulorrhaphy. Hip Int. 2013;23:576-582.

25. Garabekyan T, Chadayammuri V, Pascual-Garrido C, MeiDan O. All-arthroscopic ligamentum teres reconstruction with graft fixation at the femoral head-neck junction. Arthrosc Tech. 2016;5:e143-e147.

26. Lindner D, Sharp KG, Trenga AP, Stone J, Stake CE, Domb BG. Arthroscopic ligamentum teres reconstruction. Arthrosc Tech. 2012;2:e21-e25.

27. Amenabar T, O'Donnell J. Arthroscopic ligamentum teres reconstruction using semitendinosus tendon: Surgical technique and an unusual outcome. Arthrosc Tech 2013;1:e169-e174.

28. Mei-Dan O, McConkey MO. A novel technique for ligamentum teres reconstruction with "all-suture" anchors in the medial acetabular wall. Arthrosc Tech 2014;3:e217-e221. 